\title{
A CAPACITOR DISCHARGE, QUASI-TRAPEZOIDAL PULSE GENERATOR FOR PARTICLE EXTRACTION
}

\author{
J. Bonthond and E.B. Vossenberg - CERN, Geneva, Switzerland
}

\begin{abstract}
$\underline{\text { Abstract }}$
In the CERN SPS accelerator two methods for particle extraction are used. One of these methods, called Slow Extraction, delivers extracted beams with a duration of up to several seconds to the majority of experiments. The other one, the Fast Resonant Extraction, providing particle bursts with a duration of a few milliseconds, is used for neutrino experiments. For the latter kind of extraction a quadrupole magnet is installed, which is connected to a high voltage pulse generator delivering quasi-trapezoïdal current pulses. The pulse generator is a capacitor discharge system generating current pulses, with a rising slope having 2 different gradients, of which the second one is approximately zero.The falling slope is obtained through natural decay in a freewheel circuit. The use of modern GTO (Gate Turn Off) power switches resulted in a much simpler circuit than the use of standard thyristors would have permitted.
\end{abstract}

\section{Introduction}

When a charged capacitor is discharged into an inductance, from the moment onwards the discharge switch is closed, an oscillation starts between the capacitor and the inductance. In the generator described in chapter 2.1, the oscillation is interrupted before the top of the first half-period. From the moment of interruption onwards the inductor current decays through a freewheel circuit. In this way, with an appropriate component selection, a quasi-triangular current waveform can be obtained.

This kind of pulse generator was originally used for Fast Resonant Particle Extraction in the CERN SPS accelerator. Basically Fast Resonant Extraction was obtained by exiting a quadrupole magnet with a current pulse having the quasi-triangular waveform (Ref. 1). Herewith the beam was pushed onto resonance at the rising slope of the pulse and after a few milliseconds the resonance was stopped at the falling slope. During resonance particles, resonating with a sufficiently high amplitude in the horizontal plane, enter the extraction channel and leave the SPS. The "spill" (i.e. the number of particles extracted per time interval) of a beam extracted with a triangular current pulse had an approximately Gaussian structure. Because a more rectangular spill structure is better suited for the experiments, a generator delivering current pulses with a quasi-trapezoïdal waveform was developed (Ref. 2). By introducing a resistor in the discharge circuit during the oscillation period, the rising current slope can be changed. With appropriate resistor selection a modified slope with near zero gradient can be obtained.

Thereafter new operational requirements for 2 independently adjustable pulses per SPS cycle demanded an upgrading of the generator into a double pulsing system (Ref. 3). The availability of modern semiconductor power switches, in this case GTO devices, has greatly simplified the original circuit, equipped with standard thyristors which have the disadvantage that switching off is only possible with forced commutation. 


\section{Basic Circuit Description}

2.1. Triangular pulse shape

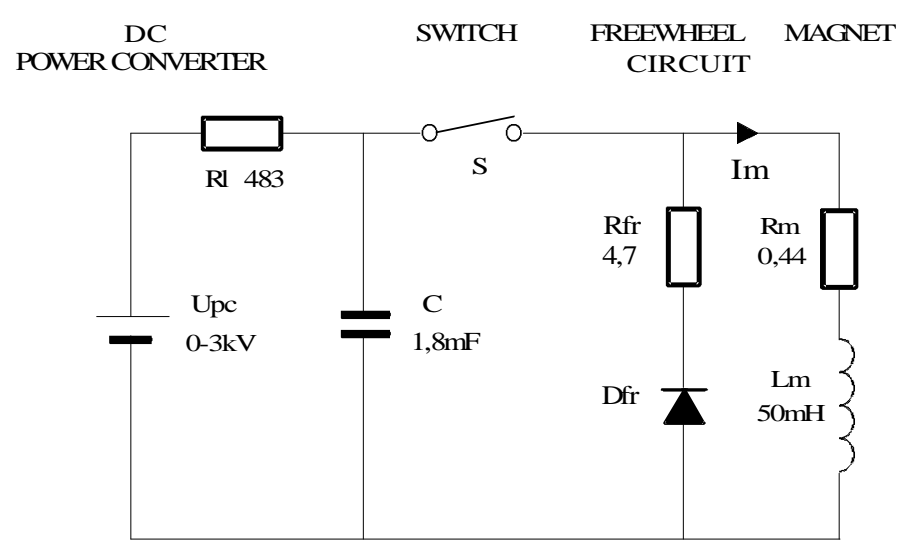

Figure 1. Original circuit - Triangular pulse shape

Capacitor $\mathrm{C}$ is charged with a regulated DC power converter $U_{p c}$ to the required positive voltage via $R_{l}$. At the moment, the extraction has to start, switch $\mathrm{S}$ is closed and a current $I_{m}$ starts to flow from capacitor $\mathrm{C}$ into the magnet $\left(L_{m}, R_{m}\right)$.

The magnet current is a damped sinewave :

$I_{m}=\frac{e^{-\delta t}}{\sqrt{1-\xi^{2}}} \cdot \frac{U_{0}}{Z_{0}} \cdot \sin \omega t$ with $\delta=\frac{R_{m}}{2 L}, \xi=\frac{R_{m}}{2 Z_{0}}, Z_{0}=\sqrt{\frac{L}{C}}$ and $\omega \cong \omega_{0}=\sqrt{\frac{1}{L C}}$

With $\omega \cong \omega_{0}$ the half-period time being $\cong 30 \mathrm{~ms}$, the factor $e^{-\delta t}(\approx 0,94$ at $\mathrm{t}=15 \mathrm{~ms})$ has only a small influence since switch $\mathrm{S}$ will normally open before the top of the first half-period.

Because of the polarity of the freewheel diode $D_{f r}$, no current can flow during this phase into the freewheel circuit $\left(D_{f r}, R_{f r}\right)$. At the moment the extraction has to stop, switch $\mathrm{S}$ opens and the magnet current $I_{m}$ is interrupted. Thereby the magnet voltage reverses and the freewheel diode $D_{f r}$ starts to conduct. $I_{m}$ then decays with a time constant $: t_{d}=\frac{L_{m}}{R_{f}}$

The resulting current waveform is an asymmetrical quasi-triangle.

The amplitude of its maximum can be adjusted by the capacitor voltage and the moment of its maximum by the timing of switch $\mathrm{S}$.

\subsection{Trapezoïdal pulse shape}

As was mentioned in the introduction, the spill structure can be improved with a rising slope having two different gradients, the second one being approximately zero. 


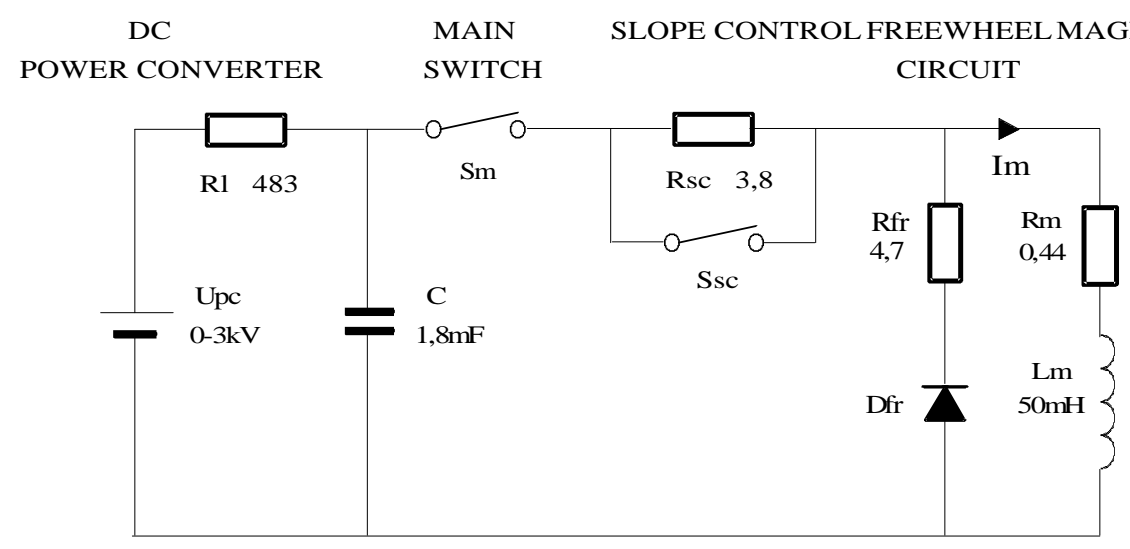

Figure 2. Modified circuit - Trapezoidal pulse shape

The required change of gradient is realized by means of a series resistor $R_{s c}$ (nominally 3,8 Ohm) in the main current path. During the first part of the slope, the resistor is short-circuited with an auxiliary switch (Slope Control) and the gradient of the slope is determined by sin $\omega t$ as was mentioned before. At the moment the gradient has to change the Slope Control switch is opened. The magnet current now becomes :

$I_{m}=e^{-\delta_{s} \cdot \Delta t} \cdot \frac{U_{c(s)}}{Z} \cdot\left\{\left(\frac{Z}{\omega_{s} \cdot L}-\frac{\delta_{s}}{\omega_{s}}\right) \sin \omega_{s} \cdot \Delta t+\cos \omega_{s} \cdot \Delta t\right\}$

with $\delta_{s}=\frac{R_{m}+R_{s c}}{2 L}, Z=Z_{0} \cdot\left(\xi+\sqrt{1-\xi^{2}} \cdot \operatorname{ctg} \omega t_{s}\right), \xi=\frac{R_{m}}{2 Z_{0}}$ and $\omega_{s}=\sqrt{\omega_{0}^{2}-\delta_{s}^{2}}$

\section{Practical calculations}

Maximum voltage : $U=3 k V$

Capacitor value $\quad: C=1,8 m F$

Magnet data $\quad: L=50 \mathrm{mH} ; R=440 \mathrm{~m} \Omega$

$Z_{0}=\sqrt{\frac{L}{C}}=\sqrt{\frac{5 \cdot 10^{-2}}{1,8 \cdot 10^{-3}}} \cong 5,27 \Omega, \delta=\frac{R}{2 L}=\frac{440 \cdot 10^{-3}}{2 \cdot 50 \cdot 10^{-3}}=4,4$ and

$\omega_{0}=\frac{1}{\sqrt{L . C}}=\frac{1}{10^{-3} \sqrt{50.1,8}} \cong 105 \rightarrow \omega \cong \omega_{0}=105 \rightarrow t=\frac{2 \pi}{\omega} \cong \frac{2 \pi}{105} \cong 59,8 \mathrm{~ms}$

$I_{M A X}=\frac{e^{-\delta t}}{\omega L} \cdot U_{0}$ and $I_{M A X}$ occurring at $\frac{t}{4} \cong 15 m s \quad \rightarrow \quad e^{-\delta t}=e^{-4,4 \cdot 0,015} \cong 0,94$

Therefore $\quad I_{M A X}=\frac{0,94}{105 \cdot 50 \cdot 10^{-3}} \cdot 3 \cdot 10^{3} \cong 537 A$ 
The RMS current for a triangular waveform with duration $t$ and repetition period $\mathrm{T}$, is approximately : $I_{R M S} \cong I_{M A X} \sqrt{\frac{t}{3 T}}$

For $t \cong 60 m s$ and $T=10 s$ this gives : $I_{R M S}=537 \sqrt{\frac{60.10^{-3}}{3.10}} \cong 24 \mathrm{~A}$

\section{Circuit Simulations}

Circuit simulations for the QECD generator have been done with PSpice TM. All plots have been made at the maximum operation voltage of $3000 \mathrm{~V}$.
An important conclusion from the lower plot is that the Main switches will have to withstand approximately $4800 \mathrm{~V}$. With the selected freewheel resistor the maximum negative backswing of the magnet voltage is $1800 \mathrm{~V}$. Therefore when both channels are operating at $3000 \mathrm{~V}$, the negative backswing voltage on one channel will generate voltage of approximately $4800 \mathrm{~V}$ over the switches of the other channel. The Slope Control switch is bypassed by a low value resistor. Therefore the Main switch as to withstand practically the entire voltage.

Figure 3. Waveform simulations

\section{Switches}

As was mentioned above, GTO thyristors have been selected for the Main and Slope Control switches. The results from the circuit simulations mentioned earlier having shown that Main switch 2 has to withstand 4800V. At the moment of purchase the best available GTO devices had a rating of $4500 \mathrm{~V}$. Therefore a solution with two devices in series was chosen. The Slope Control switch unit is of the same construction as the Main switch unit but with an air cooled 3,8 $\Omega$ resistor added to it. The basic circuit of a Main switch unit is given in the following figure. As can be seen, both GTO devices have a snubber network (C1, D1, R1 and C2, D2, R2) and an antiparallel diode (D3 and D4). 


\section{Figure 4. Main Switch circuit}

\section{Legend :}

MF-Mains Filter ; TIM-Timing Fiber Optic Receiver ; GTR-Gate Trigger Unit TR-Isolation Transformer ; PS-Power Supply

\section{Generator}

The circuit diagram of the generator is shown in the next figure.

The generator is housed in two groups of 19" electronics racks. One group of 5 racks for the power electronics and a second group of 3 racks for the control electronics.

Figure 5. Generator circuit 
The control electronics are not covered by this paper.

A sparkgap, limiting the voltage across the freewheel resistor, has been added for protection in case of malfunctioning.

In the original system the addition of filter circuits at the output was found to be necessary for suppression of a parasitic oscillation at the opening of the Main switch.

The same filters are installed in the upgraded system.

\title{
7. Measurement result
}

\author{
In this plot the \\ magnet current is \\ shown in normal \\ working conditions \\ with $1500 \mathrm{~V}$ \\ operating voltage \\ and $7 \mathrm{~ms}$ slope \\ control time. \\ Vertical scale : \\ $50 \mathrm{~A} / \mathrm{div}$ \\ Horizontal scale : \\ $2 \mathrm{~ms} / \mathrm{div}$
}

\section{References :}

1. Generation of current pulses of quasi triangular shape with fast power thyristors by V. Rödel, G.H. Schröder, E.B.Vossenberg. CERN SPS 80-05

2. Half - Integer Resonant Extraction with Quasi Rectangular Spill by Marcel Gyr and Eugène B. Vossenberg. CERN SL 92-15

3. The QECD pulse generator for Fast Resonant Extraction by Jan Bonthond.

CERN SL 94-5 